\title{
FEASIBILITY OF A SMALL, RAPID OPTICAL/IR RESPONSE, NEXT GENERATION GAMMA-RAY BURST MISSION
}

\author{
B. Grossan ${ }^{1,2}$, G.F. Smoot ${ }^{1,4}$, V.V. Bogomolov ${ }^{1}$, S.I. Svertilov ${ }^{1}$, \\ N.N. Vedenkin ${ }^{1}$, M. Panasyuk ${ }^{1}$, B. Goncharov ${ }^{3}$, G. Rozhkov ${ }^{3}$, \\ K. Saleev ${ }^{3}$, E. Grobovskoj ${ }^{1}$, A.S. Krasnov ${ }^{1}$, V.S. Morozenko ${ }^{1}$, \\ V.I. Osedlo ${ }^{1}$, E. Rogkov ${ }^{1}$, T.V. Vachenko ${ }^{1}$ and E.V. Linder ${ }^{1,2}$
}

\begin{abstract}
We present motivations for and study feasibility of a small, rapid-optical/IR response gamma-ray burst (GRB) space observatory. By analyzing existing GRB data, we give realistic detection rates for X-ray and optical/IR instruments of modest size under actual flight conditions. Given new capabilities of fast optical/IR response $(\sim 1 \mathrm{~s}$ to target) and simultaneous multi-band imaging, such an observatory can have a reasonable event rate, likely leading to new science. Requiring a Swift-like orbit, duty cycle, and observing constraints, a Swift-BAT scaled down to $190 \mathrm{~cm}^{2}$ of detector area would still detect and locate about 25 GRB $\mathrm{yr}^{-1}$ for a trigger threshold of $6.5 \sigma$. About $23 \%$ of $\mathrm{X}$-ray located GRB would be detected optically for a $10 \mathrm{~cm}$ diameter instrument ( $\sim 6 \mathrm{yr}^{-1}$ for the $6.5 \sigma \mathrm{X}$-ray trigger).
\end{abstract}

\section{Introduction}

Swift has been spectacularly productive in the study of gamma-ray bursts (GRBs), but is past its design lifetime. A new GRB observatory with new capabilities would be welcome. No obvious replacement is on the horizon: the SVOM mission is now uncertain, and other upcoming observatories described as "GRB-capable" lack: (i) high GRB rates, (ii) an on-board optical instrument, and (iii) optical-quality positions. Without (i), you cannot do statistical studies; without (ii) \& (iii) you cannot apply ever-evolving techniques in follow-up observations that make Swift so productive. Here we study a small post-Swift GRB observatory with the following

\footnotetext{
1 Extreme Universe Laboratory, Moscow State University, Russian Federation

2 University of California at Berkeley Space Sciences Laboratory, USA

3 Moscow State University, Russian Federation

4 University of California at Berkeley, USA
} 
requirements: small, due to limited resources in the current world economy; high GRB rate, to enable statistical studies; new capabilities, to investigate new science; optical quality locations, to enable the most varied possible follow-up science. Is this feasible? How small could a GRB observatory instrument really be?

\section{The GRB rate for a "mini-BAT"}

From Beppo-Sax to Swift-BAT, coded mask X-ray cameras have yielded high GRB rates \& localizations smaller than optical telescope fields. In Burrows et al. (2012), the wide field of view of coded mask cameras dominated energy range \& sensitivity for maximum GRB rate. Consider a BAT scaled down in detector area, but with the same field of view (FOV), for GRB location Such a scaled-down BAT can still achieve a relatively high detection rate: for steady sources, rate depends on $\mathrm{SNR}$ (signal to noise ratio), $\mathrm{SNR} \sim \mathrm{A}^{1 / 2}$ for steady sources, and so rate must be weakly dependent on detector area, A. GRBs are transient, however; their detected light curve, fluence \& duration change with background noise, therefore A, so the SNR relation can be more complex. We therefore used actual BAT light curves to predict performance of a smaller BAT, to determine how small an instrument would still locate GRBs at a high rate.

\subsection{Method: Rate estimate from BAT data $S N R_{\text {peak }}$ measurements}

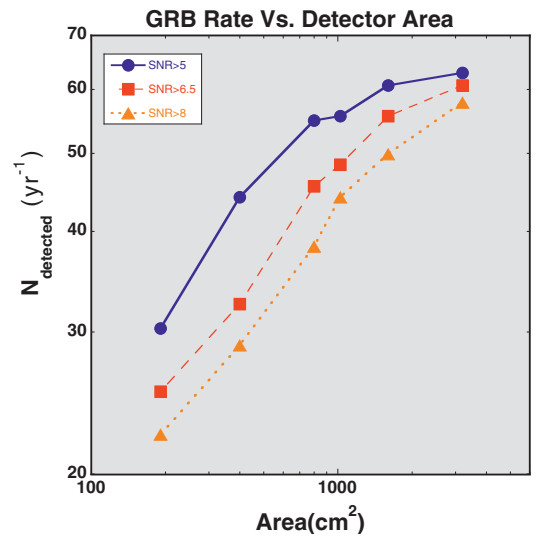

Fig. 1. GRB Detection Rate vs. Detector Area for Swift BAT-Like instruments.
The peak SNR time segment of a GRB light curve determines the smallest instrument collecting area, $\mathrm{A}_{\text {collect }}$ required for its detection. GRB rate as a function of $\mathrm{A}_{\text {collect }}$ was determined by (i) finding the peak SNR segment in BAT light cuves, (ii) scaling to get $\operatorname{SNR}_{\text {peak }}\left(A_{\text {collect }}\right)$, then (iii) counting the number of bursts with $\mathrm{SNR}_{\text {peak }}>$ threshold to determine the detection rate.

We used a very simple $\mathrm{SNR}_{\text {peak }}$ "Trigger", specifed as follows: We used the sum of $64 \mathrm{~ms}$ data channels 1-3 (15-100 kev, the highest S/N combination). Integration time windows of $0.25,0.5,1,2,4,8 \mathrm{~s}$ were examined for fluctuations $>$ threshold (in $\sigma$ ) over background (the trigger and detection criteria). The trailing average background ( $\mathrm{t}-19.2$ to $\mathrm{t}-6.4 \mathrm{~s}$ ) was used. All triggers were checked by eye for false triggers (only 1 found). (BAT also has a long time window image data trigger, rarely triggered; we had no such trigger, as the benefit for a small instrument would likely be very small.) We analyzed 94 GRB light curves 2010 Nov. - 2012 Mar to find the SNR peak in each window. We then scaled 
the SNR for instruments of smaller collecting area, and reported the number of bursts over trigger threshold in the smaller instruments.

Pre-selecting only burst data, as we did, begs the question of false alarm rate. This pre-selection is acceptable because in a real mision, known tools are available to control excessive false alarms: trigger parameter tuning, cutoff rigidity maps, and others.

\subsection{Analysis results}

Our simplified trigger detected $91 \%$ of BAT bursts (86 detections, 1 fail, 7 image trigger non-detects). For only $190 \mathrm{~cm}^{2}$ of collecting area, $<1 / 25$ th of Swift, $22 \mathrm{GRB} / \mathrm{yr}$ would still have $\mathrm{SNR}_{\text {trig }}>8$; this number increases to 25 and 30 for $\mathrm{SNR}_{\text {trig }}$ of 6.5 and 5.0 , respectively ${ }^{5}$.

Imaging/location consistent with triggering analysis. After triggering, an image is made, with location uncertainty $\sim 1 / \mathrm{SNR}_{\text {image. }}$ Is the correlation noise of coded-mask imaging, the dominant noise in $\mathrm{SNR}_{\text {image }}$, a problem? In a simulation by Connell (2012), all triggers with $\mathrm{SNR}_{\text {peak }}>5$ yielded $\mathrm{SNR}_{\text {image }}>8$, the typical coded mask design threshold. Localization quality is therefore not a problem.

Robust result. We recognize this approach is valid only for BAT-like instruments with similar orbit, operations \& observing constraints. Background depends on instrument \& spacecraft construction, via activation \& secondary emission. The BAT background is $\sim 1.9$ cts s $^{-1} \mathrm{~cm}^{-2} 15-150 \mathrm{keV}$ (bat_desc.html). The ESA MXGS coded mask camera, with similar CZT detectors (5 mm thick) and shielding, on the International Space Station (copious mass \& solid angle for secondaries), has estimated background $=2.1 \mathrm{cts} \mathrm{s}^{-1} \mathrm{~cm}^{-2} 15-200 \mathrm{keV}$ (Renzi 2009). The results are therefore not sensitive to spacecraft platform; orbital inclination and altitude dominate the background considerations.

\section{New science from follow-up optical/IR}

A "mini-BAT" would sample the brightest of the known Swift GRB population. How then, do we get new science? Swift optical follow-up is hardwarelimited to $>60 \mathrm{~s}$ after trigger. Telescope pointing has been achieved with beamsteering mirrors in $\sim 1$ s over similar sized fields by the upcoming Ultra-fast Flash

\footnotetext{
${ }^{5}$ The Ultra-Fast Flash Observatory-pathfinder X-ray camera (UFFO-p; Kim et al. 2012) has $190 \mathrm{~cm}^{2}$ collecting area, but is planned to fly in a polar orbit ( $89^{\circ}$ inclination) with high background regions, losing substantial useful observing time. We roughly estimate a duty cycle of $20 \%$ of that of BAT (from the time in high background regions and a $1000 \mathrm{~s}$ background decay after).

From this, and a field of view $84 \%$ that of BAT's, we find that UFFO-p's X-ray rates are $\sim 0.17$ of those above, $\left(4.3 \mathrm{yr}^{-1}, \mathrm{SNR}_{\text {trig }}=6.5\right)$ assuming all else identical to BAT. We then expect $\sim 1$ detection $\mathrm{yr}^{-1}$ for the $10 \mathrm{~cm}$ UVOT-like optical instrument on UFFO-p (see Sect. 3.1).
} 
Observatory-Pathfinder (Jeong et al. 2012). Such rapid-response on-orbit optical follow-up would take advantage of the shorter communication time and lack of weather of ground-based rapid follow-up. Such an instrument would yield new information: optical or IR bulk Lorentz factors from the time of opt/IR peak (Molinari et al. 2007); a much better sample of GRB optical rise times (these rise times are often less than the $>60 \mathrm{~s}$ Swift UVOT response, and so are infrequently measured); the first dust evaporation detection via time-resolved simultaneous multi-band colors (all of which occurs in $<60 \mathrm{~s}$, too fast for most telescopes; see Grossan et al. 2012 for details \& additional science topics).

We did not consider a focused X-ray telescope (XRT) for a small observatory, as such instruments are large, expensive, \& complex. An IR/optical imager would still yield a precise position for its detections, partially replacing an XRT.

\subsection{Aperture size vs. rate}

The brightest Swift UVOT V fluxes for each GRB (Fig. 2) show a detection rate strongly dependent on sensitivity. Sensitivity, dominated by non-instrument background, scales as 1/aperture diameter, D (for same exposure time and pixel size). For typical conditions, rate decreases by a factor of 0.8 for $2 \mathrm{X}$ smaller D (blue line); by 0.7 for a $3 \mathrm{X}$ smaller D (pink line). For a 190 (or 1000) $\mathrm{cm}^{2}$ mini-BAT, Swift orbit \& constraints, the GRB Rate plot (Fig. 1), gives 25 (or 48) GRB X-ray locations/yr (6.5 $\sigma$ trigger). Conservatively neglecting any correlation of $\mathrm{V} v s$. $\mathrm{X}$, for $\mathrm{D}_{\mathrm{UVOT}} / 2$, $\sim 7$ (or 13) optical detections/yr are predicted. However, these rates can be sig-

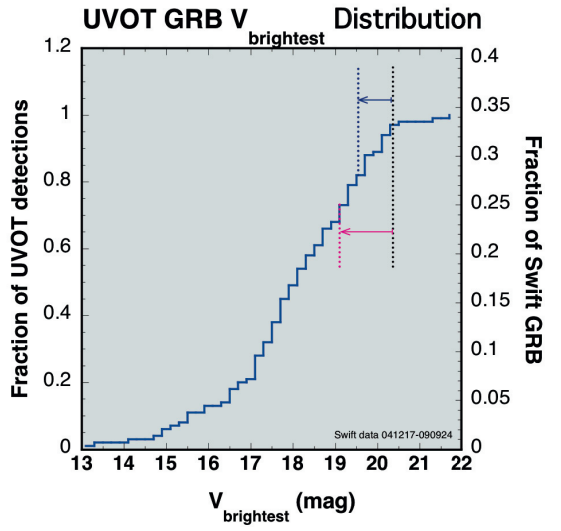

Fig. 2. Swift UVOT Maximum Brightness vs. Rate.

nificantly increased. Earlier optical acquisition may catch many bursts when brighter. A near-IR detector will get up to $50 \%$ more extinguished GRB (Perley et al. 2009). Detector quantum efficiency (QE) improvement will also boost rate (a CCD has $\sim 4-5 \mathrm{X}$ UVOT's QE).

\section{Discussion \& summary}

Our conservative analysis using Swift data firmly supports the feasibility of small GRB missions: small instruments with good orbits and high duty cycle can produce GRB locations at useful rates for follow-up studies. Improving on Swift technology can boost these rates by improving detector sensitivity: e.g., SVOMlike $5-150 \mathrm{keV}$ detectors give $\sim 2.7 \mathrm{X}$ BAT $(15-150 \mathrm{keV})$ source photon flux. Better optical QE and IR sensitivity would increase optical/IR rates. 
Rapid acquisition for prompt optical emission enables new lines of inquiry. An additional IR channel would yield the first prompt IR measurements and permit the study of dynamic extinction. Additional bands would give more information for small mass cost.

Smaller X-ray cameras can roughly measure GRB durations \& spectra, but will have poor Short GRB rates, and greater uncertainty in $\mathrm{E}_{\text {peak }}$ and flux. We find that new science, even limited to the brightest GRBs of the already known Swift population, outweighs these disadvantages. In the future, we will extend this work to investigate the performance of more types of X-ray, optical, IR detectors, spacecraft, and orbits.

We gratefully acknowledge the support of the Russian Federation Ministry of Education and Science, Agreement No. 11.G34.31.0076.

\section{References}

bat_desc.html: http://heasarc.nasa.gov/docs/swift/about_swift/bat_desc.html Burrows, D.N., Fox, D., Palmer, D., et al., 2012, Mem. Suppl. Soc. Astron. Ital., 21, 59 Connell, P., 2012, presentation, this conf., and priv. comm.

Grossan, B., Park, I.H., Ahmad, S., et al., 2012, SPIE Astronomical Telescopes + Instrumentation (Amsterdam RAI, The Netherlands, July 1-6), 8443, 84432R

Jeong, S., Ahmad, S., Barrillon, et al., 2012, SPIE Astronomical Telescopes + Instrumentation (Amsterdam RAI, The Netherlands, July 1-6), 8443, 84432S

Kim, J.E., Ahmad, S., Barrillon, P., et al., 2012, SPIE Astronomical Telescopes + Instrumentation (Amsterdam RAI, The Netherlands, July 1-6), 8443, 84432V

Molinari, E., Vergani, S.D., Malesani, D., et al., 2007, A\&A, 469, 13

Perley, D.A., Cenko, S.B., Bloom, J.S., et al., 2009, AJ, 138, 1690

Renzi, F., 2009, 6th GEANT 4 Space Users Workshop, Madrid (http://www. inta.es/g4suw2009/docs/Presentations/20_Wed_May_2009/G4SUWS_2009_Renzi_ BackgroundEstimationInMXGS.pdf) 
